\title{
Síndrome Congénito de Klippel-Trenaunay-Weber. Caso Clínico
}

\author{
Congenital Klippel-Trenaunay-Weber Syndrome. Clinical Case
}

\author{
${\text { Gabriela Espín }{ }^{1} \text {; Leslie Suntaxi²; Cristina Yambay²; Richard Silva }{ }^{3,4} \text {; Luis Espín }{ }^{3,4} \text { \& Bélgica Vásquez }}^{5}$
}

\begin{abstract}
ESPÍN, G.; SUNTAXI, L.; YAMBAY, C.; SILVA, R.; ESPÍN, L. \& VÁSQUEZ, B. Síndrome congénito de Klippel-TrenaunayWeber. Caso clínico. Int. J. Morphol., 38(6):1842-1848, 2020.

RESUMEN: El síndrome de Klippel-Trenaunay-Weber (SKTW) es una enfermedad congénita poco frecuente caracterizada por hipertrofia de un miembro que afecta a los huesos y a las partes blandas, con extensos angiomas planos, várices y otras anomalías vasculares, como fístulas arteriovenosas. Su incidencia es de 1:100.000 personas. El objetivo fue describir un caso raro de SKTW bilateral, confirmado con hallazgos clínicos e imagenológicos en un niño de 9 años de edad, de sexo masculino, con antecedentes de sangrado digestivo bajo, herniorrafía inguinal izquierda y orquidopexia ipsilateral. En ambos miembros inferiores se identificaron lesiones hemangiomatosas e hipertrofia muscular y edema en miembro inferior izquierdo con ausencia de segundo y tercer dedos del pie izquierdo por antecedente quirúrgico de amputación. Adicionalmente, presentaba adenopatías cervicales e inguinales. Dentro de los hallazgos radiográficos importantes, se observó una cortical ósea delgada en el fémur del miembro inferior izquierdo. El SKTW afecta típicamente a los miembros inferiores de forma unilateral; este es un caso infrecuente de afección bilateral (con predominio izquierdo). Algunos pacientes, registran compromiso visceral con hemorragia digestiva baja, además de alteraciones genitourinarias, presentes en el $30 \%$ de los casos. La presentación de adenopatías es inusual.
\end{abstract}

PALABRAS CLAVE: Síndrome de Klippel-Trenaunay-Weber; Hipertrofia osteomuscular; Anormalidades vasculares.

\section{INTRODUCCIÓN}

El Síndrome de Klippel-Trenaunay-Weber (SKTW) es una rara enfermedad congénita que afecta el desarrollo de vasos sanguíneos, tejidos blandos y huesos (Lacerda et al., 2014). Fue nombrado así por los médicos franceses Maurice Klippel y Paul Trenaunay, quienes lo describieron por primera vez en 1900 (Barbe, 2019). Posteriormente, en 1918 Parkes Weber describió un paciente con los tres signos precedentes más las fístulas arteriovenosas en el miembro afectado (Servelle, 1985). Tiene una prevalencia desconocida y se han reportado 1000 casos en todo el mundo, su incidencia es aproximadamente 1:100.000 nacidos vivos (Lorda-Sanchez et al., 1998); no muestra predilección por ningún sexo o ancestría; y los casos familiares se han observado con poca frecuencia (Lacerda et al.). La etiología del SKTW es aún desconocida y la mayoría de los casos son esporádicos por mutaciones somáticas, especialmente del gen PIK3CA (Barbe). Así, se propone que el SKTW, en lugar de ser una entidad clínica distinta, pertenece al espectro de los sobrecrecimientos relacionados con PIK3CA ("PIK3CA-related overgrowth spectrum" o PROS); sin embargo, se necesita más investigación para confirmar la causa (Babe; Vahidnezhad et al., 2016).

Hasta ahora se han notificado menos de 30 mutaciones distintas en el gen PIK3CA en PROS, y se ha demostrado que cinco de ellas son recurrentes. Un estudio reciente identificó cualquiera de estas cinco mutaciones en 20 de los 21 casos de SKTW (Keppler-Noreuil et al., 2015; Vahidnezhad et al.). Esto lleva a la activación de la AKT, y esta vía conduce a un aumento de la proliferación celular a través de la mTOR1, con un sobrecrecimiento tisular clínicamente reconocible (Vahidnezhad et al.). Dentro de sus diagnósticos diferenciales se indican al Síndrome de Parkes-Weber, Síndrome de Proteus y Síndrome de Cloves (Rahimi et al., 2017).

El cuadro clínico consiste en la tríada de angiomas cutáneos de color vino oporto, varicosidades e hipertrofia de los tejidos blandos y óseos de la región afectada, que suele tener predominio unilateral y de miembros inferiores (Rahimi et al.; Escobar \& Ayra, 2019).

\footnotetext{
${ }^{1}$ Servicio de Ortopedia y Traumatología del Hospital de Especialidades Fuerzas Armadas No 1 , Quito, Ecuador.

${ }^{2}$ Universidad Central del Ecuador, Facultad de Ciencias Médicas, Quito, Ecuador.

${ }^{3}$ Servicio de Ortopedia y Traumatología Pediátrica del Hospital de Especialidades Baca Ortiz, Quito, Ecuador,

${ }^{4}$ Universidad Central del Ecuador, Quito, Ecuador.

${ }^{5}$ Universidad de Tarapacá, Arica, Chile.
} 
Una vez identificado al paciente clínicamente, se le debe someter a estudios de imagen con la finalidad de evaluar alteraciones vasculares, musculares y óseas. En muchos casos, la clínica es variable, detectando anomalías rotacionales en los miembros afectados, escoliosis en columna y, entre de las complicaciones, se pueden presentar hemorragias, tromboflebitis $(20-45 \%)$, fístulas arteriovenosas, fallo cardiaco congestivo, tromboembolia pulmonar (4 - $25 \%$ ), hematuria, hemorragia gastrointestinal, estreñimiento y obstrucción del ostio interno de la uretra, hipoplasia linfática $(50 \%)$, linfoedema y celulitis a repetición (Hernández et al., 2017).

Por su bajo costo, se recomienda la realización de ecografía Doppler como estudio inicial y flebografía con contraste para evaluar neoformaciones vasculares y linfáticas. Se debe someter al paciente a estudio radiográfico para evaluar longitud ósea, tamaño de corticales y existencia de acortamientos o angulaciones en los miembros afectados. La angiorresonancia se cataloga como un estudio adecuado para descartar afección vascular intraabdominal y pelviana; la resonancia magnética ayuda a evaluar hipertrofia o cambios en tejidos de los miembros afectados (Chimbo et al., 2018).

Como el SKTW se presenta con poca frecuencia y su manifestación clínica es variable, no se dispone de mucha información sobre su manejo, pero al comprometer varias estructuras vasculares, linfáticas, músculo esqueléticas, dérmicas y tejidos blandos, el manejo debe ser multidisciplinario, y el tratamiento es principalmente sintomático y conservador. Actualmente se han descrito estudios con terapia farmacológica antitumoral, como Sirolimus (Rapamicina), para retardar la proliferación de las células musculares lisas y del endotelio vascular; Bleomicina, ya que actúa como esclerosante, mostrando resultados satisfactorios en malformaciones linfáticas y tratamiento con láser, indicado en los hemangiomas y úlceras presentes (Gupta, 2018; Neira et al., 2014).

En cuanto a tratamientos más invasivos de tipo quirúrgico, se aplican cuando hay presencia de úlceras y síntomas moderados o graves, con complicaciones de insuficiencia venosa, fallo cardíaco, coagulopatías de consumo, en los que se opta por terapia láser endovenosa, embolización para malformaciones arteriovenosas o uso de chips de plata para interrumpir la comunicación venosa (Gontero et al., 2017; Rahimi et al.). En los casos de alteraciones osteomusculares, de acuerdo a cada caso, se debe evaluar si existen acortamientos en las miembros, deformidades angulares o incapacidades funcionales, debiendo realizar manejos ortopédico o quirúrgico dependiendo del grado de malformación y discapacidad que presente el paciente (Rahimi et al.). En algunos casos, la patología se puede presentar con macrodactilia, en la cual se debe aplicar resección radial, siendo el principal objetico, obtener un pie no doloroso y funcional. La amputación del dedo, la epifisiodesis y reducción de los tejidos blandos, acompañado de una resección parcial o total de la falange distal, y la amputación en rayo (pie de princesa), son aplicados en los casos en que el miembro afectado causa alteración motriz (Chimbo et al.; Gupta).

A continuación, describimos un caso muy raro de SKTW bilateral, confirmado con hallazgos clínicos e imagenológicos.

\section{CASO CLÍNICO}

Paciente de 9 años de edad, de sexo masculino, consultó al Servicio de Ortopedia Pediátrica para valoración del miembro inferior izquierdo por aumento de tamaño y cambio de coloración. Además, referenció presentar dolor en pies y tobillos al caminar, con gran dificultar para saltar y dolor predominante en la zona lumbar.

Los antecedentes clínicos revelaron que a los tres años de edad fue diagnosticado con SKTW. A los 23 días de nacido, fue intervenido quirúrgicamente por herniorrafía inguinal izquierda y orquidopexia ipsilateral. Al año de nacido, presentó abscesos en la región inguinal izquierda y entre los tres y cuatro años, abscesos bilaterales en la región glútea. Por presentar macrodactilia progresiva, a los dos años de edad, fue sometido a amputación radial del segundo y tercer dedos del pie izquierdo, ya que no podía utilizar calzado y su bipedestación era dolorosa por híper presión digital. A los tres años de edad fue sometido a resección de quistes dermoides en el muslo izquierdo y de tejidos redundantes en la pierna izquierda. Presentó sangrado digestivo bajo a los cuatro años, hemorroides a los seis años y celulitis recurrente en el miembro inferior izquierdo entre los cuatro y ocho años de edad.

En el examen físico se evidenció incremento de masa muscular en el miembro inferior izquierdo y edema no doloroso, masa dolorosa de $2 \mathrm{~cm}$ de diámetro en la cara posterior del muslo derecho, lesiones bilaterales tipo hemangiomas y angioqueratomas, ausencia del segundo y tercer dedos del pie izquierdo por antecedente quirúrgico con formación de muñón en condición adecuada (Fig. 1); acortamiento de 2 $\mathrm{cm}$ del miembro inferior izquierdo, con test de Galeazzi positivo por diferencia a nivel del fémur (Fig. 2); la anisomelia fue compensada o sin marcha en Trendelenburg. A nivel de columna se observó hiperlordosis (Fig. 3) y desviación de columna lumbar con ligera rotación y compensa- 
ción pélvica. Se apreció adenopatías cervicales, retroauriculares, suboccipitales e inguinales de menos de 1 $\mathrm{cm}$. También presentaba eczema atópico en pliegues, además de marcada tortuosidad vascular izquierda en escroto y aparente atrofia testicular.

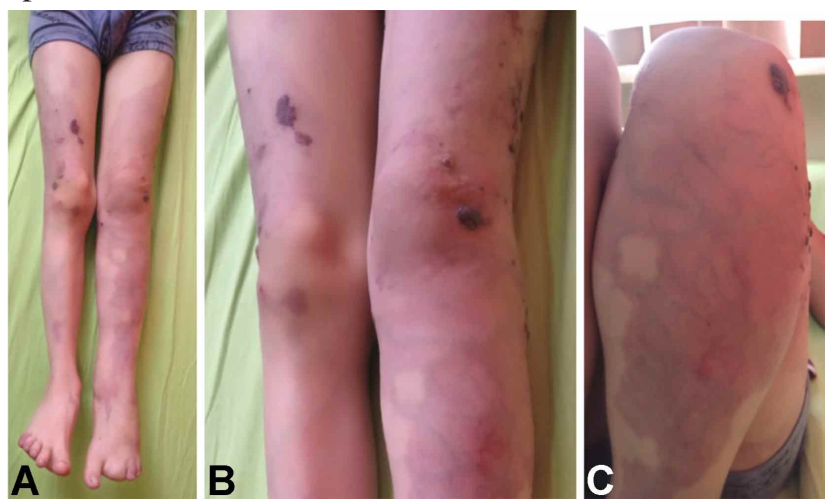

Fig. 1. Miembros inferiores con grandes angiomas cutáneos bilaterales de color vino porto e hipertrofia de tejidos blandos en miembro izquierdo (compatible con SKTW).

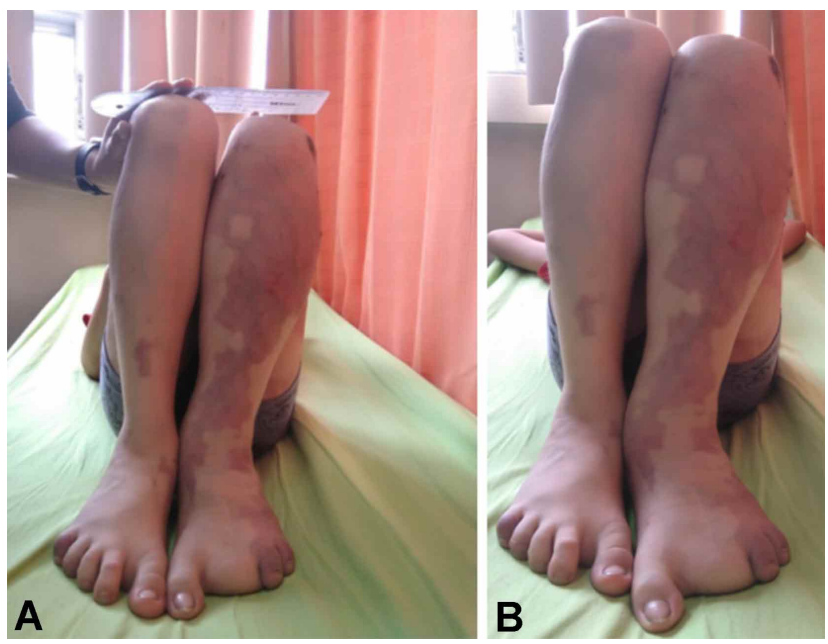

Fig. 2. Test de Galeazzi positivo por acortamiento de $2 \mathrm{~cm}$ en miembro inferior izquierdo por acortamiento del fémur.

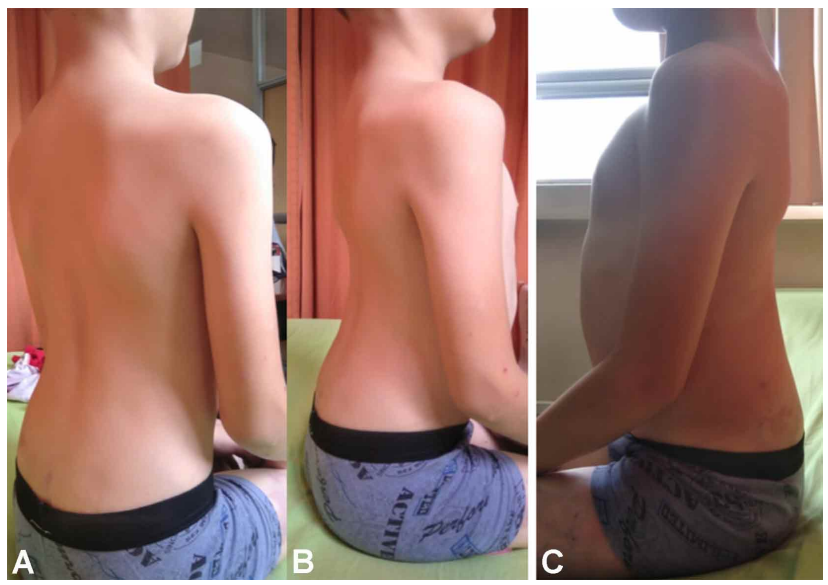

Fig. 3. El examen físico de columna vertebral reveló un aumento de la curva lumbar fisiológica.
En estudios de imagen, la radiografía anteroposterior de columna toracolumbar evidenció escoliosis con desviación de curvatura dorso lumbar hacia la izquierda en $15^{\circ}$ de Cobb, con compensación pelviana (Fig. 4). En radiografías anteroposterior lateral y axial de ambos fémur, se evidenció disminución de las corticales en el miembro inferior izquierdo en comparación con el derecho y menor tamaño de la cabeza femoral. El fémur derecho se apreció con angulación cervicodiafisaria de $130^{\circ}$ y el fémur izquierdo con angulación cervicodiafisaria de $153^{\circ}$, con acortamiento de $1,5 \mathrm{~cm}$ y desviación en valgo con antecurvatum; también se observó aumento de la musculatura y presencia de calcificaciones musculares en pierna izquierda (Fig. 5). En radiografías anteroposterior y lateral de ambas piernas, se evidenció disminución de la longitud de la tibia izquierda en comparación con la derecha y disminución del grosor cortical de la tibia y fíbula izquierda. La tibia derecha presentó un ángulo fémoro-tibial anatómico de $6^{\circ}$ en valgo y la tibia izquierda un ángulo fémoro-tibial anatómico de $10^{\circ}$ en valgo (Fig. 6). En radiografías anteroposterior y oblicua del pie izquierdo se evidenció ausencia de segundo y tercer rayo (segundo hueso metatarsiano y falanges y parte distal del tercer hueso metatarsiano y falanges) en el pie derecho no se observaron alteraciones (Fig. 7).

En la resonancia magnética del miembro inferior izquierdo, se identificó aumento de la trama vascular y vasos linfáticos y presencia de varices, las fibras musculares presentaron áreas de tendinosis y tenosinovitis (Fig. 8).

La ecografía testicular mostró malformaciones vasculares escrotales de predominio izquierdo, características atróficas en testículo izquierdo y alteración de la ecogenicidad de manera bilateral. La ecografía de cuello evidenció glándulas tiroideas y paratiroideas normales, con
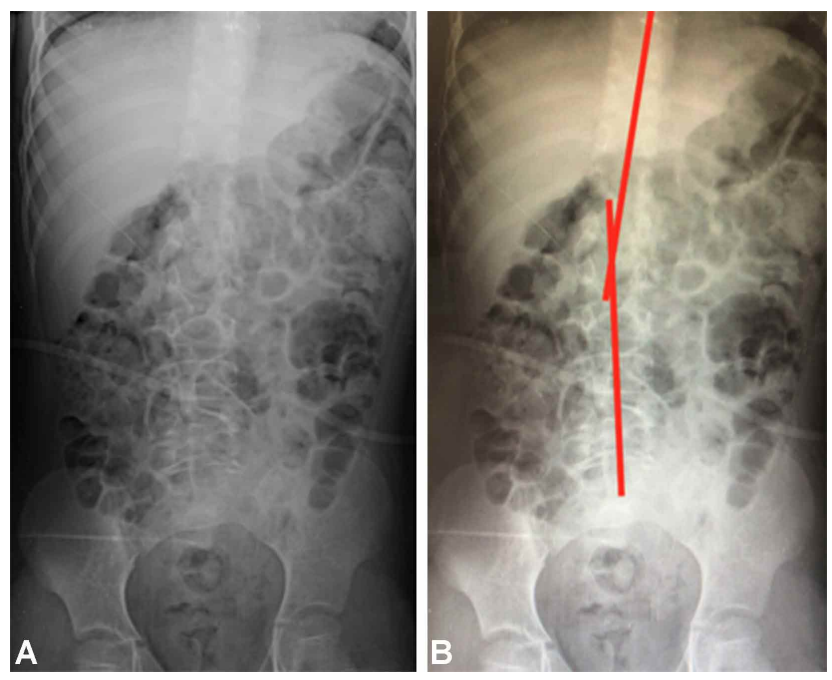

Fig. 4. Escoliosis de $15^{\circ}$ a nivel de T11- T12 con curvatura tóracolumbar izquierda. 


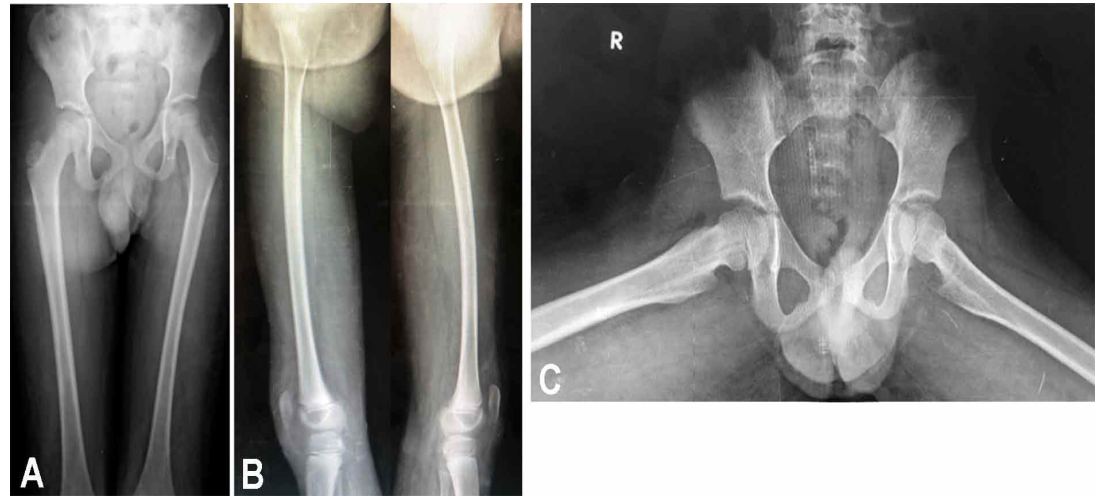

Fig. 5. Cabeza femoral pequeña del fémur izquierdo. Corticales diafisarias delgadas con antecurvatun, acortamiento y valgo femoral, en comparación con miembro inferior derecho.

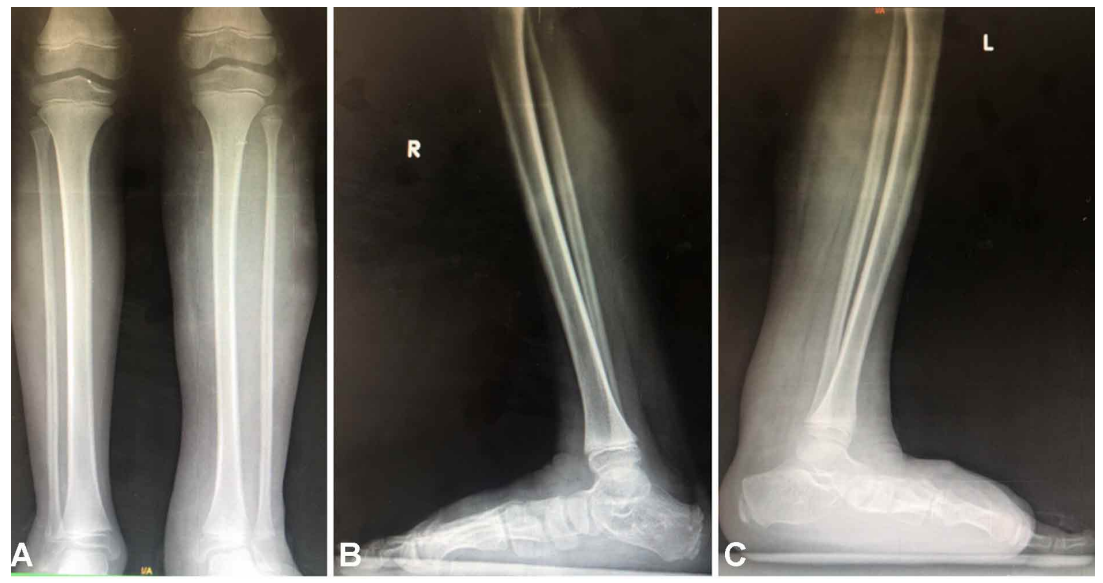

Fig. 6. Rx antero posterior y lateral de ambas piernas. Se evidencia disminución en el tamaño de la tibia izquierda en comparación a la derecha; disminución en el grosor cortical de la tibia y fíbula del lado izquierdo. Tibia derecha con ángulo fémoro-tibial anatómico de $6^{\circ}$ y tibia izquierda con ángulo fémoro-tibial anatómico de $10^{\circ}$ en valgo.
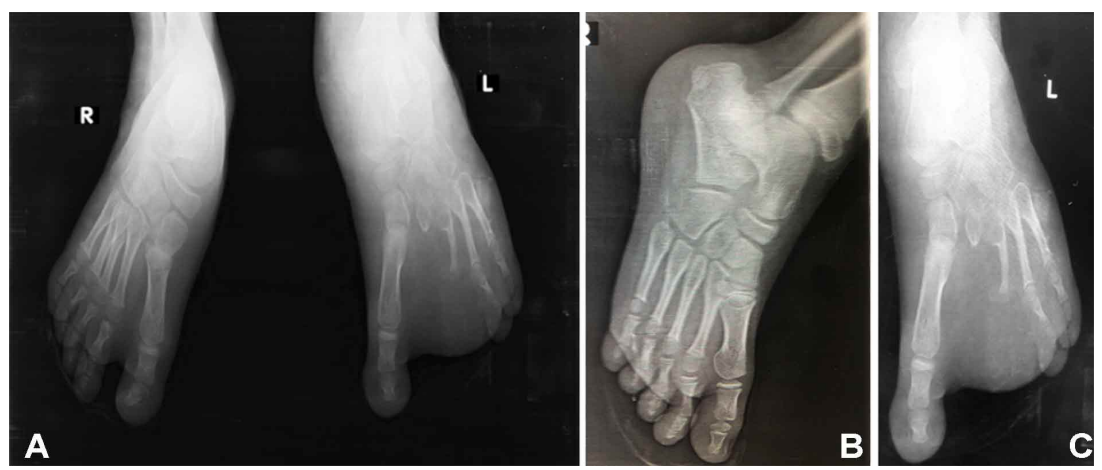

Fig.7. Radiografía AP de ambos pies. Se observa ausencia del segundo metatarsiano y parte del tercer metatarsiano y de los dedos II y III, en pie izquierdo.

adecuada vascularización y presencia de nódulos linfáticos IIB bilaterales. La vena yugular interna y la arteria carótida común del lado derecho presentaron signos inflamatorios con un diámetro de $0,8 \mathrm{~cm}$. La ecografía del muslo izquierdo evidenció la presencia de una masa hiperecogénica a nivel del tercio medio de la cara posteromedial; dilatación aneurismática trombosada subaguda, de lesiones vasculares ya conocidas por patología de base del paciente; también se evidenció permeabilidad del eje arterial del miembro inferior derecho y dificultad para visualizar la vena femoral en tercio medio del muslo. La ecografía venosa de los miembros inferiores reveló la presencia y permeabilidad del sistema venoso profundo y superficial con áreas de insuficiencia. Los exámenes de biometría hemática, química sanguínea, electrolitos y dímero $\mathrm{D}$, se encontraron dentro de rangos normales.

\section{DISCUSIÓN}

El SKTW, es una enfermedad de baja prevalencia que se agrupa en el decimoséptimo capítulo de la lista de códigos CIE-10, dentro de los síndromes de malformaciones congénitas que afectan principalmente a los miembros (Q87.2); esta categoría también incluye a los síndromes de: Holt-Oram, patela en uña, Rubinstein-Taybi, sirenomelia, trombocitopenia con ausencia de radio, VATER (OPS, 1995). El Ministerio de Salud Pública de Ecuador lo clasificó como la octava enfermedad catastrófica dentro de las enfermedades raras o infrecuentes. Hernández et al. el año 2017 detectaron y estudiaron 23 pacientes diagnosticados con fístulas arteriovenosas congénitas (SKTW) a través de exámenes clínicos y estudios complementarios en Ecuador.

El SKTW se presenta con más frecuencia en miembros inferiores y es más común de forma unilateral, con un $85 \%$, siendo bilateral solamente en un $12,5 \%$, dejando el $2,5 \%$ restante a la forma bilateral cruzada (Redondo et al., 2011). En el caso del paciente reportado, se evidenció compromiso bilateral con predominio izquierdo, en el cual se identificó signos como Galeazzi positivo, aumento del volumen del muslo y disminución, tanto en grosor cortical, como en longitud ósea del miembro inferior izquierdo, caso similar al descrito por Abril et al. (2019). Se sospecha que la escoliosis o desviación lateral de la 

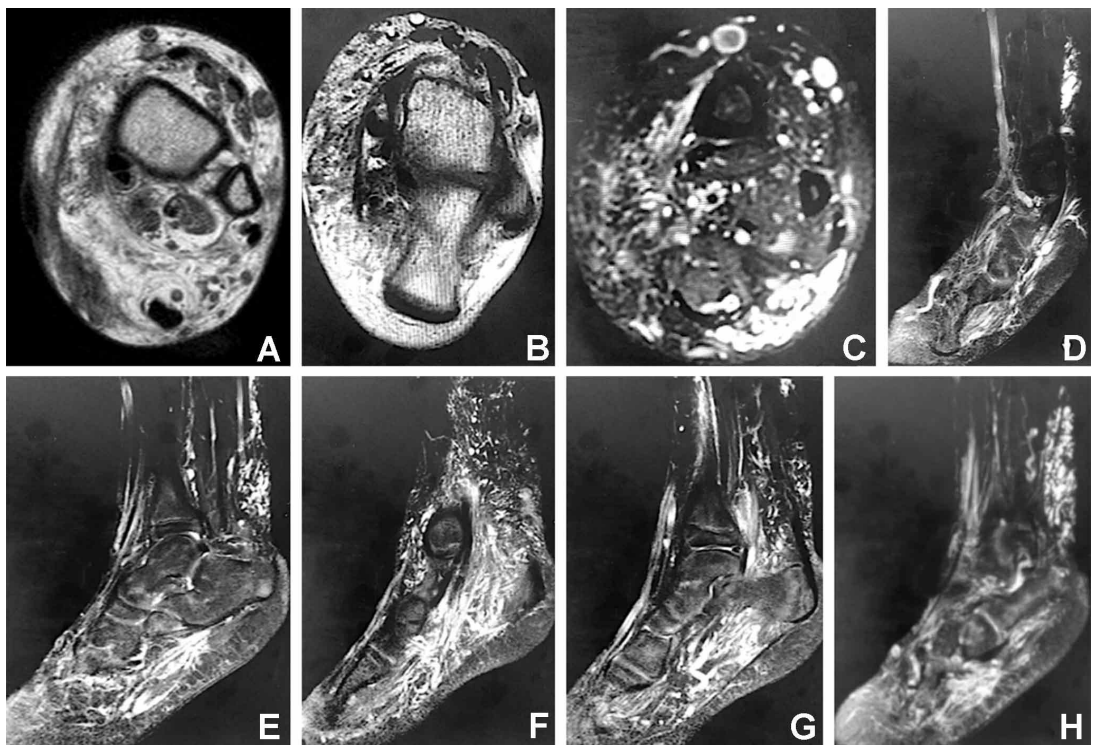

Fig. 8. Resonancia magnética de miembro inferior izquierdo. Se observa alteración vascular con aumento en tamaño de los vasos, aumento de masa muscular, presencia de nodulación en región posterior al tendón calcáneo y áreas tendinosas con cambios hipertróficos.

columna, relacionada con el SKTW, fue producida por la mala angulación del eje motor del miembro inferior izquierdo, para compensar dicha asimetría durante la marcha. Al parecer, estas alteraciones, como lo señaló Bueno Sánchez, (2014), producen hiperlordosis lumbar de manera compensatoria.

Dentro de los hallazgos radiográficos importantes, se observó corticales delgadas en el fémur del miembro inferior izquierdo. Según el esquema de clasificación de Dorr (Dorr et al., 1993), en base al grosor del hueso cortical y la forma del canal medular, el paciente presentó el tipo morfológico B y respecto a la conformación proximal del fémur, el lado izquierdo presentó un índice de Dorr menor. Además, por la pérdida de tejido óseo, el miembro inferior izquierdo presentó tendencia a la deformidad angular en valgo y con antecurvatum.

Los aportes vasculares anómalos pueden provocar áreas de trabeculación escasa y/o prominente (Moreno et al., 2004), en nuestro caso las alteraciones vasculares provocaron disminución del flujo en el miembro afectado del paciente. Es importante considerar que, debido a las malformaciones vasculares, cualquier procedimiento ortopédico en pacientes con SKTW debe ser evaluado cuidadosamente. Debido a la lenta tasa de curación que presenta este grupo de pacientes, Nahas et al. (2014) recomiendan un manejo conservador y ortopédico y en caso de decisión quirúrgica, tomar en consideración el aumento del riesgo de hemorragia.
El compromiso vascular gastrointestinal ha sido descrito hasta en un $20 \%$ de los casos, principalmente en colon sigmoide y recto y durante la primera década de vida con carácter intermitente (Sepulveda et al., 2018; Wang et al., 2010). De acuerdo a lo señalado por Gandhi et al. (2019) los pacientes con sangrado clínicamente significativo pueden requerir resección intestinal segmentaria.

Husmann et al., 2007 reportaron que en el $30 \%$ de los casos, los pacientes con SKTW presentan alteraciones genitourinarias; en nuestro caso, el paciente presentaba malformaciones vasculares escrotales y atrofia testicular izquierda. Sin embargo, no observamos malformaciones vasculares cutáneas en el área genital como lo observaron Husmann et al. en el $7 \%$ de los casos.

Pocos estudios se han centrado en la participación del sistema linfático en el SKTW, aunque algunas evidencias sugieren que las anomalías linfáticas están asociadas con la enfermedad. Al respecto, Liu et al. (2010) encontraron hiperplasia de vasos linfáticos en el $35 \%$, hipoplasia o aplasia en el $65 \%$ y linfedema en el $100 \%$ de los miembros afectadas. Yamaki et al. (2013) encontraron malformaciones linfáticas extratronculares en el $21 \%$, de estos pacientes el $20 \%$ tenían malformaciones linfáticas infiltrantes difusas, mientras que sólo el $2 \%$ tenía lesiones localizadas. Por el contrario, se encontraron malformaciones linfáticas trunculares en el $28 \%$, lesiones obstructivas en el $8 \%$ y lesiones dilatadas en el $20 \%$. Nuestro caso presentó malformaciones linfáticas con adenopatías cervicales y adenopatías inguinales.

Nag et al. (2018) evaluaron la eficacia y seguridad de la escleroterapia para malformaciones vasculares pediátricas en 61 pacientes. Demostraron que la escleroterapia con agentes a base de espuma reduce eficazmente los síntomas con una tasa aceptable de complicaciones. No obstante, señalaron que se necesitan más estudios para determinar los agentes esclerosantes óptimos para subconjuntos individuales de malformaciones vasculares en la población pediátrica.

En los últimos años se ha demostrado que el manejo temprano, dentro de las dos semanas después del nacimiento, con láser de colorante pulsado se puede tratar el SKTW con un menor riesgo de fracaso y de manera más eficaz. 
Según Rahimi et al. para las lesiones refractarias se recomiendan las opciones terapéuticas más invasivas como ablación térmica endovenosa selectiva, extracción quirúrgica, flebectomía y reconstrucciones venosas profundas.

En cuanto a las angulaciones en valgo del miembro inferior izquierdo y la escoliosis, y al ser nuestro paciente pediátrico que aún se encontraba en desarrollo, se decidió realizar control de angulación ortopédico mediante férulas y fisioterapia para la corrección de la angulación, además del uso de plantilla de soporte para mejorar la carga plantar en el pie izquierdo.

\section{CONCLUSIONES}

El SKTW es una patología rara e infrecuente y no tiene cura. Debe ser manejada multidisciplinariamente por varios servicios como cirugía vascular, pediatría, cirugía ortopédica pediátrica, cardiología, cirugía general, dermatología y psicología. Se debe valorar al paciente y hacer un adecuado seguimiento de su desarrollo, ya que los cambios vasculares pueden alterar estructuras osteomusculares y órganos. Se deben realizar pruebas diagnosticas complementarias que brinden el soporte para manejar las complicaciones clínicas. El tratamiento clínico sintomático es la base, por lo que el tratamiento quirúrgico se debe realizar solo en caso de complicaciones o síntomas graves.

ESPÍN, G.; SUNTAXI, L.; YAMBAY, C.; SILVA, R.; ESPÍN, L. \& VÁSQUEZ, B. Congenital Klippel-Trenaunay-Weber syndrome. Clinical case. Int. J. Morphol., 38(6):1842-1848, 2020.

SUMMARY: Klippel-Trenaunay-Weber syndrome (KTWS) is a rare congenital disease characterized by limb hypertrophy affecting bones and soft tissues, with extensive flat angiomas, varicose veins, and other vascular abnormalities, such as arteriovenous fistulas. Its incidence is 1: 100,000 people. The aim of this work was to describe a rare case of bilateral KTWS, confirmed with clinical and imaging findings in a 9-year-old male, with a history of lower gastrointestinal bleeding, left inguinal herniorrhaphy and ipsilateral orchidopexy. In both lower limbs, hemangiomatous lesions and muscle hypertrophy were identified, and in the left lower limb edema was identified with absence of the second and third toes due to a surgical history of amputation. Additionally, the patient presented cervical and inguinal lymphadenopathy. Among the important radiographic findings, in the left lower limb femur a thin bony cortex was observed. KTWS typically affects the lower limbs unilaterally; this is an infrequent case of bilateral affection (predominantly left). Some patients have visceral involvement with lower gastrointestinal bleeding, as well as genitourinary alterations, present in $30 \%$ of cases. The presentation of lymphadenopathy is unusual.

KEY WORDS: Klippel-Trenaunay-Weber syndrome; Osteomuscular hypertrophy; Vascular abnormalities.

\section{REFERENCIAS BIBLIOGRÁFICAS}

Abril, J. C.; Vara, P. I.; Egea, G. R. M. Montero, D. M. Displasia del desarrollo de la cadera y trastornos ortopédicos del recién nacido. Pediatr. Integral, 23(4):176-86, 2019.

Barber, C. Rare health conditions 21: Moebius syndrome, juvenile polyposis syndrome, Klippel-Trénaunay syndrome and Milroy disease. Br. $J$. Healthc. Assist., 13(3):126-30, 2019.

Bueno Sánchez, A. M. Exploración de columna y cadera: cómo manejar la escoliosis. Rev. Pediatr. Aten. Primaria Supl., 2014(23):111-7, 2014.

Chimbo, T.; Castro, Y. \& Rizo, T. A propósito de un caso: síndrome de Klippel Trénaunay. Rev. Ecuat. Pediatr., 19(1):12-15, 2018.

Dorr, L. D.; Faugere, M. C.; Mackel, A. M.; Gruen, T. A.; Bognar, B. \& Malluche, H. H. Structural and cellular assessment of bone quality of proximal femur. Bone, 14(3):231-42, 1993.

Escobar, B. Y. \& Ayra, P. C. Síndrome de Klippel-Trenaunay en un recién nacido. MEDISAN, 23(1):121-30, 2019.

Gandhi, V. V.; Gautam, P.; Pai, N. V. \& Hegde S. Young adult with varicosities and recurrent lower gastrointestinal bleeding. Int. Surg. J., 6(8):3012-4, 2019.

Gontero, R.; Ortiz, A.; Roverano, S. \& Paira, S. Síndrome Klippel-Trenaunay: comunicación de dos casos. Rev. Arg. Reum., 28(1):42-7, 2017.

Gupta, R. Multidisciplinary approach to treatment of severe KlippelTranaunay-Weber Syndrome. Rheumatol. Curr. Res., 8(3):1000244 2018.

Hernández, C.; Gaybor, J. \& Guerra, E. Fístulas arteriovenosas congénitas en Ecuador. Rev. cuba. angiol. cir. vasc.; 18(2):167-77, 2017.

Husmann, D. A.; Rathburn, S. R. \& Driscoll, D. J. Klippel-Trenaunay Syndrome: Incidence and Treatment of Genitourinary Sequelae. J. Urol., 177(4):1244-9, 2007.

Keppler-Noreuil, K. M.; Rios, J. J.; Parker, V. E.; Semple, R. K.; Lindhurst, M. J.; Sapp, J. C.; Alomari, A.; Ezaki, M.; Dobyns, W. \& Biesecker, L. G. PIK3CA-related overgrowth spectrum (PROS): diagnostic and testing eligibility criteria, differential diagnosis, and evaluation. Am. J. Med. Genet A., $167 A(2): 287-95,2015$.

Lacerda, L.; Alves, U. D.; Zanier, J. F.; Machado, D. C.; Camilo, G. B. \& Lopes, A. J. Differential diagnoses of overgrowth syndromes: the most important clinical and radiological disease manifestations. Radiol. Res. Pract., 2014: 947451, 2014.

Liu, N. F.; Lu, Q. \& Yan, Z. X. Lymphatic malformation is a common component of Klippel-Trenaunay syndrome. J. Vasc. Surg., 52:155763, 2010

Lorda-Sanchez, I.; Prieto, L.; Rodriguez-Pinilla, E. \& Martinez-Frias, M. L. Increased parental age and number of pregnancies in Klippel-TrenaunayWeber syndrome. Ann. Hum. Genet., 62(3):235-9, 1998.

Ministerio de Salud Pública (MSP). Enfermedades catastróficas. Ministerio de Salud Pública, Gobierno de la República del Ecuador, 2019. Disponible en: https://www.salud.gob.ec/valoracion_enfermedades_ catastroficas_estado_gestacion/

Moreno, M. R. V.; Martínez, B. P.; Palazón, G. R. \& Gil, H. S. Síndrome de Klippel-Trénaunay: a propósito de un caso. Rehabilitación, 38(4):18891, 2004.

Nag, U.; Turner, M.; Gilmore, B.; Leraas, H.; Mureebe, L. \& Shortell, C. SS21. Foam Sclerotherapy for Low-Flow Vascular Malformations Is Safe and Effective in Children. J. Vasc. Surg., 67(6):e166, 2018.

Nahas, S.; Wong, F. \& Back, D. A case of femoral fracture in Klippel Trenaunay syndrome. Case Rep. Orthop., 2014:548161, 2014.

Neira, J.; Recalde, M.; Manzo, C. \& Villa, S. Síndrome de Klippel-TrénaunayWeber. A propósito de un caso. Rev. Med. FCM-UCSG, 8(4):262-6, 2014.

Organización Panamericana de la Salud (OPS). Clasificación Estadística Internacional de Enfermedades y Problemas Relacionados con la Salud (CIE-10). 10ª revisión. Vol. 1, Washington, D.C., OPS, 1995.

Rahimi, H.; Hassannejad, H. \& Moravvej, H. Successful treatment of unilateral Klippel-Trenaunay syndrome with pulsed-dye laser in a 2-week old infant. J. Laser Med. Sci., 8(2):98-100, 2017. 
Redondo, P.; Aguado, L. \& Martinez-Cuesta, A. Diagnosis and management of extensive vascular malformations of the lower limb. Part I. Clinical diagnosis. J. Am. Acad. Dermatol., 65(5):893-906, 2011.

Sepulveda, A.; Soriano, H. \& Espino, A. Gastrointestinal tract involvement in Klippel-Trénaunay syndrome. Lancet Gastroenterol. Hepatol., 3(7):518, 2018.

Servelle, M. Klippel and Trenaunay's syndrome. 768 operated cases. Ann Surg., 201(3):365-73, 1985

Vahidnezhad, H.; Youssefian, L. \& Uitto, J. Klippel-Trenaunay syndrome belongs to the PIK3CA-related overgrowth spectrum (PROS). Exp. Dermatol., 25(1):17-9, 2016

Wang, Z. K.; Wang, F. Y.; Zhu, R. M. \& Liu, J. Klippel-Trenaunay syndrome with gastrointestinal bleeding, splenic hemangiomas and left inferior vena cava. World J. Gastroenterol., 16(12):1548-52, 2010.

Yamaki, T.; Konoeda, H.; Fujisawa, D.; Ogino, K.; Osada, A.; Hamahata, A.; Nozaki, M. \& Sakurai, H. Prevalence of various congenital vascular malformations in patients with Klippel-Trénaunay syndrome. J. Vasc. Surg. Venous Lymphat. Disord., 1:187-93, 2013.
Dirección para correspondencia:

Gabriela Espín

Servicio de Ortopedia y Traumatología

Hospital de Especialidades Fuerzas Armadas $N^{0} 1$

Quito

ECUADOR

\section{E-mail: gespintrauma@hotmail.com}

Recibido : 20-5-2020

Aceptado: $30-7-2020$ 